\title{
Strategies to provide care to Non-COVID Patients in the COVID Era: An Experience from a Tertiary Care Institute in Northern India
}

\section{Surekha Kishore ${ }^{1}$, Aroop Mohanty ${ }^{2}$, Vivek Hada ${ }^{3}$, Subodh Kumar ${ }^{4}$, Rama Shankar Rath ${ }^{5}$}

${ }^{1}$ Executive Director, All India Institute of Medical Sciences Gorakhpur, Uttar Pradesh; ${ }^{2}$ Assistant Professor, Department of Microbiology, All India Institute of Medical Sciences Gorakhpur, Uttar Pradesh; ${ }^{3}$ Assistant Professor, Department of Microbiology, All India Institute of Medical Sciences Gorakhpur, Uttar Pradesh; ${ }^{4}$ Additional professor, Department of Pulmonary Medicine, All India Institute of Medical Sciences, Gorakhpur, Uttar Pradesh; ${ }^{5}$ Assistant Professor, Department of Community Medicine \& Family Medicine, All India Institute of Medical Sciences, Gorakhpur, Uttar Pradesh.

\begin{tabular}{|c|c|c|c|c|c|c|c|}
\hline Abstract & Introduction & Methodology & Results & Conclusion & References & Citation & ables / Figures \\
\hline \multicolumn{8}{|c|}{ ng Author } \\
\hline \multicolumn{7}{|c|}{$\begin{array}{l}\text { Dr. Rama Shankar Rath, Assistant Professor, Department of Community Medicine \& Family Medicine, All } \\
\text { India Institute of Medical Sciences, Gorakhpur, Uttar Pradesh - } 273008 . \\
\text { E Mail ID: } \underline{\text { ramashankar.aiims@gmail.com }}\end{array}$} & 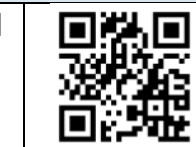 \\
\hline
\end{tabular}

\section{Citation}

Kishore S, Mohanty A, Hada V, Kumar S, Shankar R. Indian J Comm Health. 2021;33(2):404-406. https://doi.org/10.47203/IJCH.2021.v33i02.034

Source of Funding: Nil Conflict of Interest: None declared

\section{Article Cycle}

Received: 20/05/2021; Revision: 05/06/2021; Accepted: 22/06/2021; Published: 30/06/2021

This work is licensed under a Creative Commons Attribution 4.0 International License.

\section{Abstract}

With the COVID-19 pandemic the health system is facing dual burden of cases, one being the COVID-19 or Severe Acute Respiratory IIIness (SARI) cases and the second being the other Non-COVID cases. The Non-COVID cases due to the burden of the SARI cases became almost neglected by the Health System increasing the sufferings of the Non-COVID cases. In the current COVID times All India Institute of Medical Sciences, Gorakhpur continued to provide the health care to the Non-COVID cases. The current manuscript provides the detailed strategy and results of the various strategies used to provide care to such Non-COVID patients with minimum risk to the healthcare staff.

\section{Keywords:}

Humans; COVID-19; Pandemics; Government Programs

\section{Introduction}

The world is facing the destructive effects of the COVID19 pandemic since past fifteen months. $(1,2)$ Till date, 150 million cases of COVID-19 have been reported worldwide with 3 million deaths. In India the affected cases are around 20 million and total deaths 1 million deaths. It has also severely affected the health care system as well as the health of the health care personnel involved in providing care to these patients. $(3,4)$ Besides this great suffering the pandemic has further worsened the health of the NonCOVID-19 patients suffering from other acute and chronic diseases due to lack of health care facilities. $(5,6)$ We have failed miserably in providing proper health care to these Non-COVID-19 patients who tend to visit the outpatients (OPD's) for some or the other causes. According to World Health Organization, in half of the countries there is disruption to services related to diabetes and hypertension. (6) It is not only the duty of the health care system and the administration to provide care to this category of patients but also the responsibility of the same to see that they don't contract the disease while visiting the health care facility or availing health care.

This current manuscript describes the method used by a newly set up tertiary care institute in Eastern Uttar Pradesh to provide care to these group of Non-COVID-19 patients during the pandemic.

Strategy: All India Institute of Medical Sciences, Gorakhpur was established in the year 2019 to provide health care to this part of Northern India. As the institute was in its nascent stage with the inpatient facility under development, the COVID care could not be provided. It continued to render OPD services to the general public in the pandemic times. At the outset, the institute faced two main challenges first being preventing the spreading the COVID-19 infection to the patients while receiving care and second preventing the infection to the health care providers.

For this institute did the following plans:

1. Establishment of Flu desk 
2. Establishment of Flu Clinic

3. Establishment of COVID-19 testing centre

4. Screening of the staff involved in patient care and contact tracing to separate the high-risk contacts among staff.

Execution: Initially established a flu desk in the patient waiting area. The flu desk served as an initial point of contact to every patient coming to the hospital. Where every patient and the attendant accompanying the patient must visit before entering to the hospital premises. At the flu desk trained staff nurses were posted who screened the patients based on predefined symptoms and criteria. (Box-1) If the patient fulfils none of the mentioned criteria, he could visit the general treatment facility to consult his or her doctor. If the patient was found positive for any of the mentioned criteria, he / she was sent to flu clinic where a general practitioner (MBBS or above) trained in history taking and taking care of the general patients checked the patient for the symptoms, signs and history of contact with a COVID 19 patient. From the flu desk if the patient was not found to have any criteria that suggest the patient may have COVID-19 or had contact with any COVID case were again referred back to the respective department where they want to visit, and she/ he receives the treatment in the respective department for the presenting complaint similar to all COVID-19 negative patients. In case the doctor posted in the Flu clinic feels the person is either suffering from the COVID-19 or a symptomatic high risk contact then sample of the patient was taken and appropriate treatment was provided in consultation with the respective department. In case the patient is an asymptomatic one, patient was asked to report on the 6th day after the contact with the COVID-19 patient/ suspect or the day of appearance of symptom whichever is earlier. On the day of reporting the sample of the patient was taken for antigen testing by RDT, for negative patients another sample for RT-PCR was sent.

Box-1: Screening questions used at flu desk.

1. In last 24 to 48 days have you visited any place outside India?

If yes Name, the place?....

2. Are you coming from a containment zone?

3. Do you have fever?

4. Do you have following symptoms?
A. Cough
B. Shortness of breath
C. Weakness
D. Fatigue
E. Body pain
F. Running Nose
G. Loss of smell
H. Loss of taste
I. Throat pain

\section{5.}

[Strategies to provide...] | Kishore S et al

Do you have any CONTACT with any person diagnosed with COVID19 in last 14 days?

The last procedure was to keep the health care providers free from the disease. The staff and patients were advised to follow COVID appropriate behavior both inside and outside the hospital premises. If any of the staff develops any symptom, was asked to visit the flu clinic, and was tested. If the staff is asymptomatic and is a high-risk contact, then quarantined for 5 days and tested on 6th day. In case the staff was found positive he/ she was quarantined for 17 days. The contact tracing of all the positive staff was done and managed according to the following flow chart.

\section{Our Experience:}

A total of 1,23,101 patients visited the OPD's from 19th August 2020 to 30th April 2021. From these 3042 (2.5\%) patients were referred from the Flu desk to the flu clinic. The Flu clinic advised test for 2335 patients out of which 414 (17.7\%) came out to be positive for COVID-19 either by Rapid Diagnostic Tests $(297,71.7 \%)$ or by RT-PCR (117, $28.3 \%)$. A total of 36 patients were referred from the OPD's to the flu clinic. From these 36 patients, only 7 (19.4\%) were reported as positive. During the whole period of care provision i.e., 9 months, 30 health care providers and 82 support staff were found COVID-19 positive. Thus, from total of 21090 person days of service a total of 510 person days for health care providers (i.e., $2.4 \%$ ) and from 49875 person days for support staff only 1394 person days $(2.7 \%)$ were lost due to morbidity related COVID-19.

\section{Conclusion}

Providing care to all both those who are COVID-19 positive and those who are COVID-19 negative is the need of the hour. This manuscript demonstrated the method by which an infant institute managed to provide care to the nonCOVID patients in this pandemic with minimal infection transmission to the health care providers.

\section{Recommendation}

Screening of symptomatic patients, Rapid antigen testing and implementing COVID-19 appropriate behavior will help in proper segregation of non-COVID patients thus providing them with the best of health care services.

\section{Relevance of the study}

This strategy can be implemented by other health care setups thus facilitating the reopening of the Outpatient services.

\section{Authors Contribution}

SK: Manuscript review. AM: Concept, design and Manuscript preparation. VH: Concept and Design. SK: Definition of intellectual content studies. RSR: Data analysis, statistical analysis, Manuscript preparation and Manuscript editing. 


\section{Acknowledgement}

The authors acknowledge the contribution made by all members of the COVID-19 Coordination committee of AlIMS Gorakhpur.

\section{References}

1. Gupta P, Mohanty A, Narula H, Singh V, Kalita D, Varshney S, Kabi A, Aggarwal P. Concise Information for the Frontline Health care workers in the era of COVID-19. Indian J Community Health. 2020;32(2 (Supp):215-24.

2. Li Q, Guan X, Wu P, Wang X, Zhou L, Tong Y, et al. Early Transmission Dynamics in Wuhan, China, of Novel Coronavirus-Infected Pneumonia. N Engl J Med. 2020 Mar 26; 382(13):1199-207.

\section{Figures}

FIGURE 1 FLOW OF PATIENTS IN THE OUTPATIENT DEPARTMENT

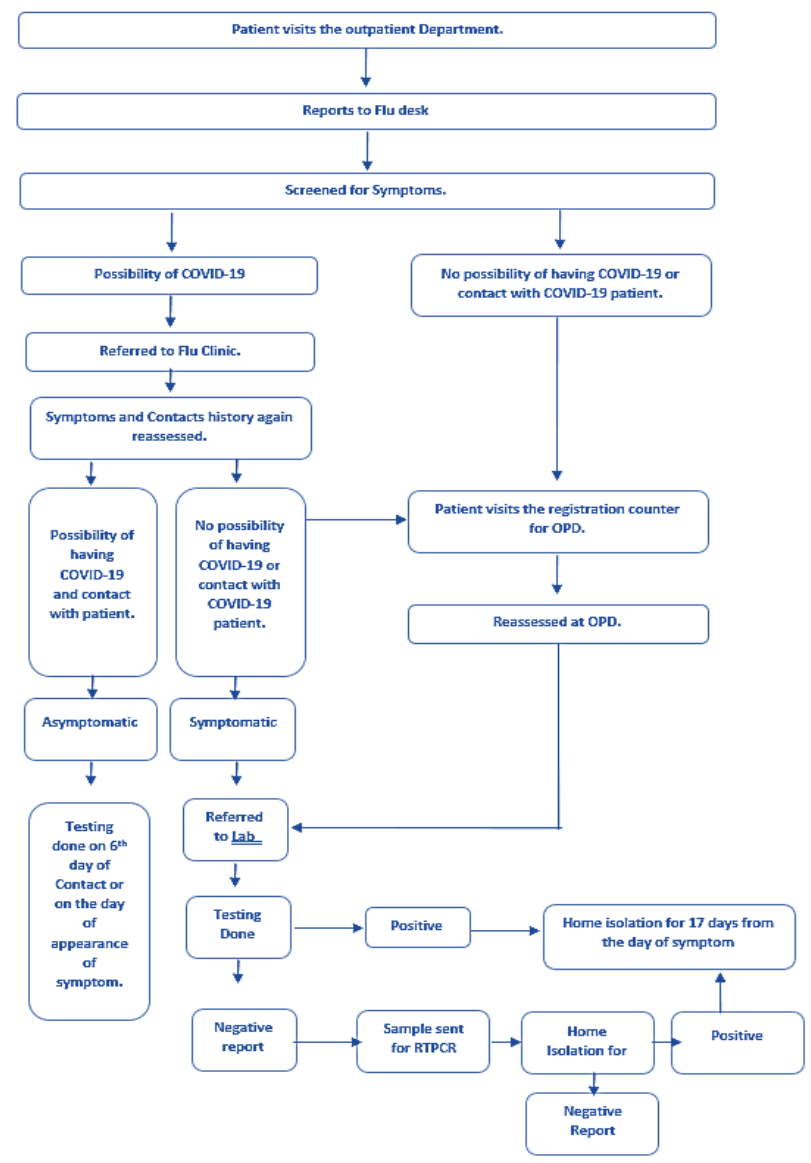

3. COVID-19: Impact on Health Care. Health Care Curr Rev. (263):2.

4. Urdaneta F, Stacey M, Sorbello M. The Adverse Impact of COVID19 on Health Care Providers: Time to Start Measuring. Anesth Analg. 2020; 131(4): e187.

5. Ahmed SAKS, Ajisola M, Azeem K, Bakibinga P, Chen Y-F, Choudhury NN, et al. Impact of the societal response to COVID-19 on access to healthcare for non-COVID-19 health issues in slum communities of Bangladesh, Kenya, Nigeria and Pakistan: results of pre-COVID and COVID-19 lockdown stakeholder engagements. BMJ Glob Health. 2020; 5(8): e003042.

6. COVID-19 significantly impacts health services for noncommunicable diseases. Available from: https://www.who.int/news/item/01-06-2020-covid-19significantly-impacts-health-services-for-noncommunicablediseases

\section{FIGURE 2 FLOW CHART FOR TESTING AND ISOLATION STRATEGY FOR HEALTH CARE PROVIDERS \& SUPPORT STAFF}

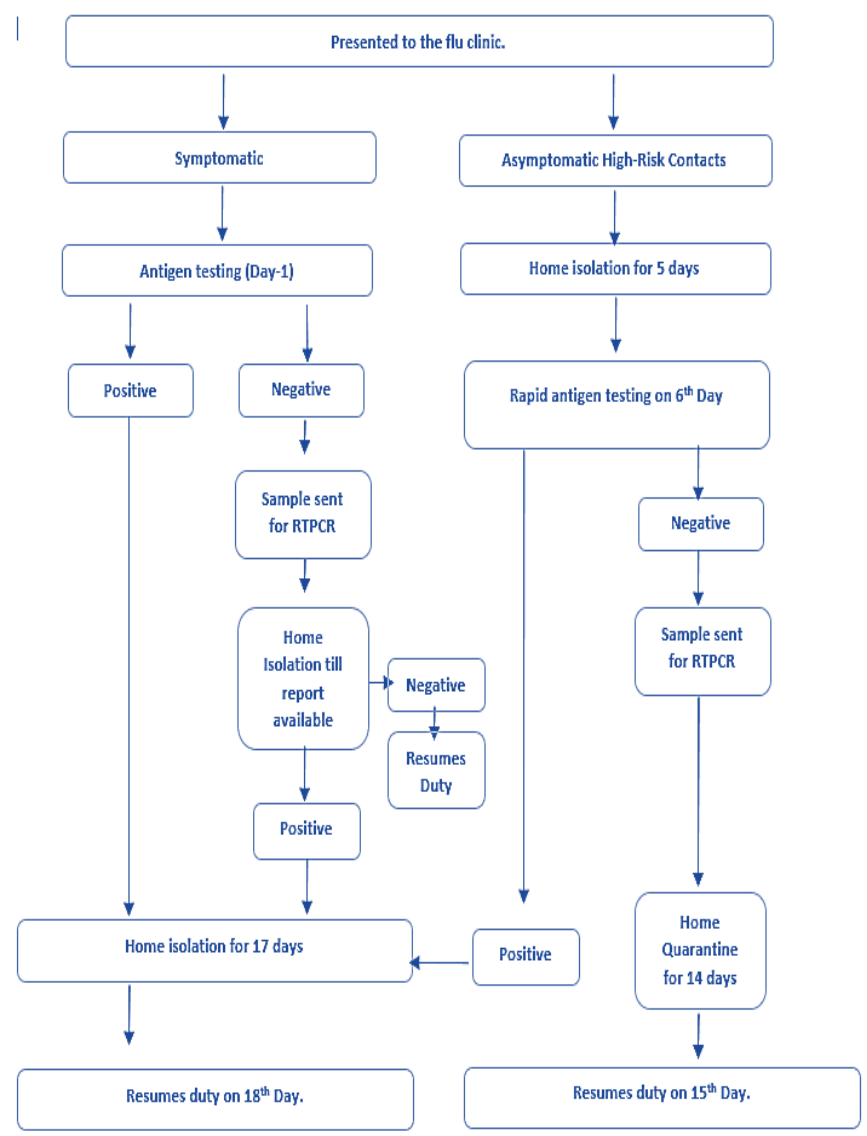

